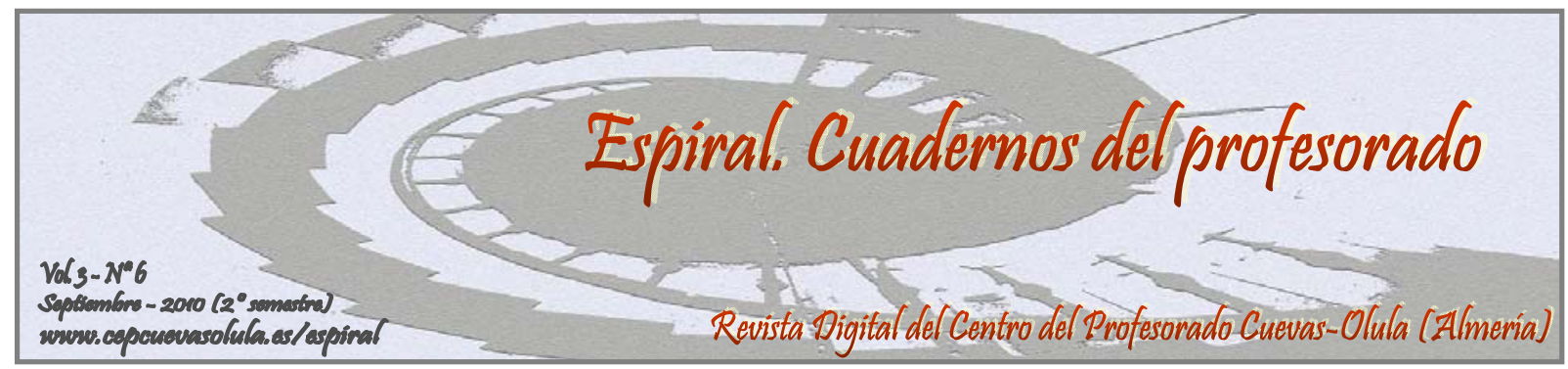

\title{
LA INTELIGENCIA EMOCIONAL Y SUS PRINCIPALES MODELOS: PROPUESTA DE UN MODELO INTEGRADOR
}

\section{EMOTIONAL INTELLIGENCE AND ITS MAIN MODELS: PROPOSAL FOR AN INTEGRATED MODEL}

\author{
Mariano García-Fernández ${ }^{(1)}$ y Sara Isabel Giménez-Mas ${ }^{(2)}$ \\ (1) IES Jaroso de Cuevas del Almanzora, Almería, España \\ ${ }^{(2)}$ Centro Educativo de Adultos, Yecla, Murcia, España
}

RESUMEN: Esta investigación ha explicado el concepto inteligencia emocional, a partir de diferentes definiciones y principios observados en la literatura. A partir de aquí, el objetivo de este trabajo es realizar un modelo integrador sobre inteligencia emocional en base a la literatura. El modelo aporta la multidisciplinariedad de uso, entre otras: educación, empresa, ONG's. Como metodología, se ha realizado una revisión de la literatura a partir de la búsqueda de datos secundarios; libros y artículos en revistas, utilizando la base de datos ABI Inform y la información referente a la inteligencia emocional recabada del seminario de inteligencia emocional, realizado por la Universidad Miguel Hernández de Elche y el sindicato ANPE. Los resultados obtenidos, explican que el modelo de inteligencia emocional propuesto, tiene dimensiones internas y externas. Las dimensiones internas son: la responsabilidad, el sentido común, la persuasión y la capacidad de aprender. Por otro lado, las dimensiones externas son: empatía, la capacidad para relacionarse, la capacidad para comunicarse, habilidad de crear modelos mentales, la voluntad, y la capacidad para adaptarse al entorno.

Palabras clave: Inteligencia emocional, modelos, aprendizaje, adaptación, TIC’s.

ABSTRACT: This research has explained the concept of emotional intelligence, using different definitions and principles observed in the literature. From here, the objective of this work is an integrative model abaut emotional intelligence based on literature. This model provides a multidisciplinary approach, including: education, business and ONG's. As a methodology, it has conducted a review of the literature from the searching of secondary data; books, articles in magazines, on ABI Inform database and information related to emotional intelligence gathered from emotional intelligence seminar, conducted by the University Miguel Hernández de Elche and the union ANPE. The results show that the proposed model of emotional intelligence has internal and external dimensions. The internal dimensions are: responsibility, common sense, persuasion and the ability to learn. On the other hand, the external dimensions are: empathy, to relate, the ability to communicate, create mental models will, and ability to adapt to the environment.

Key words: emotional intelligence, models, learning, adaptation, TIC's. 
García Fernández, M. \& Giménez-Mas, S.I. (2010). La inteligencia emocional y sus principales modelos: propuesta de un modelo integrador. Espiral. Cuadernos del Profesorado [en línea], 3(6), 43-52. Disponible en: http://www.cepcuevasolula.es/espiral.

Fecha de recepción: 14/04/2010

Fecha de aceptación: 08/06/2010
Enviar correspondencia a: elmari2001@hotmail.com

\section{1.- INTRODUCCIÓN.}

Ante los diferentes cambios de hoy día: globalización, TIC’s, los individuos y por ende las organizaciones, tienen de optar por diferentes cambios. Uno de los principales cambios es la adaptación de estos individuos a las nuevas situaciones.

La adaptación, no siempre se puede realizar de manera óptima, ya que pueden faltar habilidades o aptitudes pero podemos disminuir el gap existente, a través de la inteligencia. Ahora bien, existe un nuevo problema, qué inteligencia: inteligencia para poder observar y repetir, cociente intelectual, inteligencia abstracta... El hecho es que no es necesario tener un cociente intelectual muy alto para poder adaptarse a los cambios pero sí hace falta lo que tipificamos como inteligencia emocional. Ésta se ocupa, principalmente, de esta adaptación.

En la sociedad encontramos diversos ejemplos de ello, un alumno sin ser muy brillante en sus estudios, y a partir de su inteligencia emocional, puede obtener un puesto con un mayor status en una empresa. $\mathrm{O}$ por otro lado, un empresario o empresaria puede obtener éxito, teniendo un cociente de inteligencia menor que otro individuo que no tiene el mismo éxito con la creación de una empresa. Algún arduo lector, puede pensar que estos ejemplos también pueden tener como causa otros aspectos significativos, como por ejemplo, una mayor inversión o "estar en el momento preciso", pero no negarán que son muchos los factores que intervienen y que cabe la posibilidad de que entre uno de ellos esté la inteligencia emocional. De ahí la importancia del estudio. A partir de aquí, el objetivo de este trabajo es realizar un modelo integrador sobre inteligencia emocional en base a la literatura.

La investigación se ha estructurado en primer lugar, por medio de la metodología del estudio. A partir de aquí, se han observado las diferentes definiciones estudiadas y con la posterior propuesta de una definición propia. En tercer lugar, Se ha realizado una revisión de la literatura sobre modelos de inteligencia emocional y por último, se ha planteado un modelo, a partir de la literatura y, se han dado las conclusiones, limitaciones y futuras líneas de investigación.

\section{2.- METODOLOGÍA.}

La investigación se ha desarrollado en tres fases, ampliamente desarrolladas: una primera fase donde se recaban datos secundarios sobre inteligencia emocional, a partir de un seminario organizado por la Universidad Miguel Hernández de Elche y el sindicato ANPE, sobre inteligencia emocional y en segundo lugar, se han obtenido, así mismo, datos secundarios, a partir de la base de datos ABI Inform. De este modo, se introdujo en la base de datos las palabras inteligencia emocional, pero el registro de artículos era muy amplio, con lo cual se hizo la restricción, la de utilizar modelos de inteligencia emocional. Aún así el registro en la base de datos daba cifras demasiado elevadas para su análisis, con lo cual se acoto por fechas, desde el año 1990 hasta la actualidad. A partir de la literatura, con la obtención de estos datos secundarios, se ha reflexionado para obtener un modelo sobre inteligencia emocional.

\section{3.- DEFINICIÓN DE INTELIGENCIA EMOCIONAL.}

Existen diversas definiciones de inteligencia emocional, casi tantas como autores han escrito sobre el tema tratado. En este sentido, la inteligencia emocional es el uso inteligente de las emociones (Weisinger, 1998). Asimismo, Gardner (1993, p. 301), define inteligencia emocional como "El potencial biopsicológico para procesar información que puede generarse en el contexto cultural para resolver los problemas". Por otro lado, la inteligencia emocional se convierte en una habilidad para procesar la información emocional que incluye la percepción, la asimilación, la comprensión y la dirección de las emociones (Mayer y Cobb, 2000, p. 273). 
Mayer et al. (2000, p. 109) explicaron que la inteligencia emocional es la capacidad de procesar la información emocional con exactitud y eficacia, incluyéndose la capacidad para percibir, asimilar, comprender y regular las emociones. De este modo, la inteligencia emocional incluye las habilidades de (Mehrabian, 1996):

- Percibir las emociones personales y la de otras personas.

- Tener dominio sobre las emociones propias y responder con emociones y conductas apropiadas ante diversas circunstancias.

- Participar en relaciones donde las emociones se relacionen con la consideración y el respeto.

- Trabajar donde sea, en la medida de lo posible, gratificante desde el punto de vista emocional.

- Armonización entre el trabajo y el ocio.

Otro de los autores tratados, Bar-On (1997) define inteligencia emocional como un conjunto de capacidades, competencias y habilidades no cognitivas que influencian la habilidad propia de tener éxito al afrontar aspectos del medio ambiente. Sin embargo, uno de los principales autores, Goleman (1995b), se refiere a la inteligencia emocional como un conjunto de destrezas, actitudes, habilidades y competencias que determinan la conducta de un individuo, sus reacciones o sus estados mentales. Goleman (1995ª, p. 89) define inteligencia emocional como "capacidad de reconocer nuestros propios sentimientos y los de los demás, de motivarnos y de manejar adecuadamente las relaciones”. Más tarde Goleman (1998, p. 98), reformula esta definición de la siguiente manera:"capacidad para reconocer nuestros propios sentimientos y los de los demás, para motivarse y gestionar la emocionalidad en nosotros mismos y en las relaciones interpersonales".

Otros autores como Martineaud y Elgehart (1996, p. 48) definen inteligencia emocional como "capacidad para leer nuestros sentimientos, controlar nuestros impulsos, razonar, permanecer tranquilos y optimistas cuando no nos vemos confrontados a ciertas pruebas, y mantenernos a la escucha del otro". Asimismo, Valles (2005, p. 33) define inteligencia emocional como capacidad intelectual donde se utilicen las emociones para resolver problemas. En este sentido, la inteligencia emocional es la aptitud para captar, entender, y aplicar eficazmente la fuerza y la perspicacia de las emociones en tanto que fuente de energía humana, información, relaciones e influencia (Cooper y Sawaf, 1997, p. 52).

Una vez expuestas diferentes definiciones sobre el tema tratado, se entiende que la inteligencia emocional es una forma de interactuar con el mundo, que tiene en cuenta los sentimientos, y engloba habilidades tales como el control de los impulsos, la autoconciencia, la motivación, el entusiasmo, la perseverancia y/o la agilidad mental. Estas características configuran rasgos de carácter como: la autodisciplina, la compasión o el altruismo, que resultan indispensables para una buena y creativa adaptación social (Gómez et al., 2000, p. 55-56).

A partir de aquí, se ha realizado la siguiente definición de inteligencia emocional. Ésta es la capacidad que tiene el individuo de adaptarse e interaccionar con el entorno, dinámico y cambiante a raíz de sus propias emociones.

\section{4.- PRINCIPIOS DE LA INTELIGENCIA EMOCIONAL.}

La siguiente cuestión, una vez definida lo que es inteligencia emocional, es proporcionar unos principios básicos para que se pueda obtener una correcta inteligencia emocional. Se puede decir que la inteligencia emocional, fundamentalmente (Gómez et al., 2000) se basa en los siguientes principios o competencias:

- Autoconocimiento. Capacidad para conocerse uno mismo, saber los puntos fuertes y débiles que todos tenemos.

- Autocontrol. Capacidad para controlar los impulsos, saber mantener la calma y no perder los nervios.

- Automotivación. Habilidad para realizar cosas por uno mismo, sin la necesidad de ser impulsado por otros.

- Empatía. Competencia para ponerse en la piel de otros, es decir, intentar comprender la situación del otro.

- Habilidades sociales. Capacidad para relacionarse con otras personas, ejercitando dotes comunicativas para lograr un acercamiento eficaz.

- Asertividad. Saber defender las propias ideas no respetando la de los demás, enfrentarse a los conflictos en vez de ocultarlos, aceptar las críticas cuando pueden ayudar a mejorar. 
- Proactividad. Habilidad para tomar la iniciativa ante oportunidades o problemas, responsabilizándose de sus propios actos.

- Creatividad. Competencia para observar el mundo desde otra perspectiva, diferente forma de afrontar y resolver problemas.

Este conjunto de principios expuestos, darán lugar a una mayor o menor inteligencia emocional. En este sentido, el hecho de que un individuo pueda tener una mayor creatividad que otro individuo, no quiere decir que de forma intrínseca obtenga una mayor inteligencia emocional, ya que concurren otros factores como si el individuo sabe explotar esa creatividad. Por el contrario, la falta de creatividad se puede ver compensado por una mayor automotivación.

\section{5.- MODELOS DE INTELIGENCIA EMOCIONAL.}

A partir de la literatura, se ha realizado una revisión de los principales modelos sobre inteligencia emocional. Éstos se han clasificado en modelos mixtos, modelos de habilidades y otros modelos que complementan a ambos.

\section{1.- Modelos mixtos.}

Entre los principales autores se encuentran Goleman (1995 a y b) y Bar-On (1997). Éstos incluyen rasgos de personalidad como el control del impulso, la motivación, la tolerancia a la frustración, el manejo del estrés, la ansiedad, la asertividad, la confianza y/o la persistencia.

\section{Modelo de Goleman.}

Goleman establece la existencia de un Cociente Emocional (CE) que no se opone al Cociente Intelectual (CI) clásico sino que ambos se complementan. Este complemento se manifiesta en las interrelaciones que se producen. Un ejemplo lo podemos observar entre las comparaciones de un individuo con un alto cociente intelectual pero con poca capacidad de trabajo y otro individuo con un cociente intelectual medio y con alta capacidad de trabajo. Ambos pueden llegar al mismo fin, ya que ambos términos se complementan.

Los componentes que constituyen la IE según Goleman (1995a) son:

- Conciencia de uno mismo (Selfawareness). Es la conciencia que se tiene de los propios estados internos, los recursos e intuiciones.

- Autorregulación (Self-management). Es el control de nuestros estados, impulsos internos y recursos internos.
- Motivación (Motivation). Se explican como tendencias emocionales que guían o que facilitan el logro de objetivos.

- Empatía (Social-awareness). Se entiende como la conciencia de los sentimientos, necesidades y preocupaciones ajenas.

- Habilidades sociales (Relationship management). Es la capacidad para inducir respuestas deseables en los demás pero no entendidas como capacidades de control sobre otro individuo.

Este modelo tiene su aplicación en diferentes ámbitos como el organizacional y el laboral, este último desarrollado por el autor en su libro The Consortium for Research on Emotional Intelligence in Organizations.

\section{$\square$ Modelo de Bar-On.}

Su tesis doctoral realizada en 1988, con el nombre de: "The developmnet of a concept of psychological well-being”, constituyó la base de sus posteriores formulaciones sobre la inteligencia emocional (Bar-On, 1997) y su medida a través del inventario EQ-I (Bar-On Emotional Quotient Inventory).

El modelo está compuesto por diversos aspectos: componente intrapersonal, componente interpersonal, componente del estado de ánimo en general, componentes de adaptabilidad, componentes del manejo del estrés $\mathrm{y}$, componente del estado de ánimo en general.

Componente intrapersonal:

- Comprensión emocional de sí mismo: habilidad para comprender sentimientos y emociones, diferenciarlos $\mathrm{y}$, conocer el porqué de los mismos.

- Asertividad: habilidad para expresar sentimientos, creencias, sin dañar los sentimientos de los demás y, defender nuestros derechos de una manera no destructiva.

- Autoconcepto: capacidad para comprender, aceptar y respetarse a sí mismo, aceptando los aspectos positivos y negativos, así como las limitaciones.

- Autorrealización: habilidad para realizar lo que realmente podemos, deseamos y se disfruta.

- Independencia: capacidad para autodirigirse, sentirse seguro de sí mismo en nuestros pensamientos, acciones $\mathrm{y}$, ser independientes emocionalmente para tomar decisiones.

Componente interpersonal: 
- Empatía: habilidad para sentir, comprender y apreciar los sentimientos de los demás.

- Relaciones interpersonales: capacidad para establecer y mantener relaciones satisfactorias, caracterizadas por una cercanía emocional.

- Responsabilidad social: habilidad para mostrarse como una persona cooperante, que contribuye, que es un miembro constructivo, del grupo social.

Componentes de adaptabilidad:

- Solución de problemas: capacidad para identificar y definir los problemas y, generar e implementar soluciones efectivas.

- Prueba de la realidad: habilidad para evaluar la correspondencia entre los que experimentamos y lo que en realidad existe.

- Flexibilidad: habilidad para realizar u ajuste adecuado de nuestras emociones, pensamientos y conductas a situaciones y condiciones cambiantes.

Componentes del manejo del estrés:

- Tolerancia al estrés: capacidad para soportar eventos adversos, situaciones estresantes y fuertes emociones.

- Control de los impulsos: habilidad para resistir y controlar emociones.

Componente del estado de ánimo en general:

- Felicidad: capacidad para sentir satisfacción con nuestra vida.

- Optimismo: habilidad para ver el aspecto más positivo de la vida.

El modelo emplea la expresión "inteligencia emocional y social” haciendo referencia a las competencias sociales que se deben tener para desenvolverse en la vida. Según Bar-On (1997), la modificabilidad de la inteligencia emocional y social es superior a la inteligencia cognitiva.

\section{2.- Los modelos de habilidades.}

Son los que fundamentan el constructo de inteligencia emocional en habilidades para el procesamiento de la información emocional. En este sentido, estos modelos no incluyen componentes de factores de personalidad, siendo el más relevante de estos modelos el de Salovey y Mayer (1990). Éstos postulan la existencia de una serie de habilidades cognitivas o destrezas de los lóbulos prefontales del neocórtex para percibir, evaluar, expresar, manejar y autorregular las emociones de un modo inteligente y adaptado al logro del bienestar, a partir de las normas sociales y los valores éticos.

\section{$\square$ El modelo de Salovey y Mayer.}

El modelo ha sido reformulado en sucesivas ocasiones desde que en el 1990, Salovey y Mayer introdujeran la empatía como componente. En 1997 y en 2000, los autores realizan sus nuevas aportaciones, que han logrado una mejora del modelo hasta consolidarlo como uno de los modelos más utilizados y por ende, uno de los más populares.

Las habilidades incluidas en el modelo son las siguientes:

- Percepción emocional. Habilidad para identificar las emociones en sí mismo y en los demás a través de la expresión facial y de otros elementos como la voz o la expresividad corporal.

- Facilitación emocional del pensamiento. Capacidad para relacionar las emociones con otras sensaciones como el sabor y olor o, usar la emoción para facilitar el razonamiento. En este sentido, las emociones pueden priorizar, dirigir o redirigir al pensamiento, proyectando la atención hacia la información más importante. Por otro lado, la felicidad facilita el razonamiento inductivo y la creatividad.

- Compresión emocional. Habilidad para resolver los problemas e identificar qué emociones son semejantes.

- Dirección emocional. Compresión de las implicaciones que tienen los actos sociales en las emociones y regulación de las emociones en uno mismo y en los demás.

- Regulación reflexiva de la emociones para promover el crecimiento personal. Habilidad para estar abierto a los sentimientos ya sean positivos o negativos.

En resumen, el autor, establece una serie de habilidades internas del ser humano que ha de potenciar, en base a la práctica y la mejora continua.

\section{3.- Otros modelos.}

Incluyen componentes de personalidad, habilidades cognitivas y otros factores de aportaciones de personales, que en algunos casos son fruto de constructos creados ad hoc con la finalidad de enfatizar el sentido popular y divulgativo del constructo inteligencia emocional. 
Se le ha denominado como el modelo de los "Cuatro Pilares", por su composición. Dicho modelo se ha desarrollado, principalmente, en el ámbito organizacional de la empresa. A continuación, se han desarrollado los 4 aspectos fundamentales del modelo:

- Alfabetización emocional. Constituida por la honradez emocional, la energía, el conocimiento, el feed-back, la intuición la responsabilidad y la conexión. Estos componentes permiten la eficacia y el aplomo personal.

- Agilidad emocional. Es el componente referido a la credibilidad, la flexibilidad y autenticidad personal que incluye habilidades para escuchar, asumir conflictos y obtener buenos resultados de situaciones difíciles.

- Profundidad emocional. Se denomina a la armonización de la vida diaria con el trabajo.

- Alquimia emocional. Habilidad de innovación aprendiendo a fluir con problemas y presiones.

En este sentido Cooper y Sawaf (1997), basan la inteligencia emocional en cuatro soportes básicos. Asimismo, adapta la perspectiva de la inteligencia emocional al mundo empresarial.

\section{$\square$ El modelo de Boccardo, Sasia y Fontenla.}

Estos autores establecen las siguientes áreas, dentro del modelo en 1999:

- Autoconocimiento emocional. Reconocimiento de los sentimientos.

- Control emocional. Habilidad para relacionar sentimientos y adaptarlos a cualquier situación.

- Automotivación. Dirigir las emociones para conseguir un objetivo, en esencia para mantenerse en un estado de búsqueda permanente y mantener la mente creativa para encontrar soluciones.

- Reconocimiento de las emociones ajenas. Habilidad que construye el autoconocimiento emocional.

- Habilidad para las relaciones interpersonales. Producir sentimientos en los demás.

La principal aportación de estos autores, es la distinción entre la inteligencia emocional e inteligencia interpersonal, exponiendo que las habilidades: autoconocimiento emocional, control emocional y automotivación pertenecen a la inteligencia emocional y las capacidades: reconocimiento de las habilidades ajenas y habilidades interpersonales, forman parte de la inteligencia interpersonal.

$\square$ El modelo de Matineaud y Engelhartn.

Los autores centran su trabajo, en 1996, en la evaluación de la inteligencia emocional empleando unos cuestionarios referidos a diferentes ámbitos, integrando como componentes de la inteligencia emocional:

- El conocimiento es sí mismo.

- La gestión del humor.

- Motivación de uno mismo de manera positiva.

- Control de impulso para demorar la gratificación.

- Apertura a los demás, como aptitud para ponerse en el lugar del otro.

Este modelo se diferencia del resto por la apertura externa, introduciendo factores exógenos.

$\square$ El modelo de Elías, Tobías y Friedlander.

Estos autores señalan como componentes de la Inteligencia Emocional en 1999:

- Ser consciente de los propios sentimientos y de los demás.

- Mostrar empatía y comprender los puntos de vista de los demás.

- Hacer rente a los impulsos emocionales.

- Plantearse objetivo positivos y planes para alcanzarlos

- Utilizar habilidades sociales.

Como resumen Elias et al., (1999), integran los modelos anteriores para homogeneizar la medición del constructo inteligencia emocional.

\section{$\square$ El modelo de Rovira.}

Realiza en 1998, una valiosa aportación con respecto a las habilidades componentes de la inteligencia emocional. El autor engloba el modelo en 12 dimensiones:

I.- Actitud positiva.

- Valorar más los aspectos positivos que los negativos.

- Resaltar más los aciertos que los errores, las utilidades que los defectos, el esfuerzo que los resultados.

- Hacer uso frecuente del elogio sincero.

- Buscar el equilibrio entre la tolerancia y la exigencia.

- Ser conscientes de las propias limitaciones y de las de los demás. 
II.- Reconocer los propios sentimientos y emociones.

- Reconocer los propios sentimientos y emociones.

III.- Capacidad para expresar sentimientos y emociones.

- Expresar sentimientos y emociones a través de algún medio o cal apropiado.

IV.- Capacidad para controlar sentimientos y emociones.

- Tolerancia a la frustración.

- Saber esperar.

V.- Empatía.

- Captar las emociones de otro individuo, a través del lenguaje corporal.

VI.- Ser capaz de tomar decisiones adecuadas.

- Integrar lo racional y lo emocional.

VII.- Motivación, ilusión, interés.

- Suscitar ilusiones e interés por algo o alguien.

VIII.- Autoestima.

- Sentimientos positivos hacia sí mismo.

- Confianza en las propias capacidades para hacer frente a los retos.

IX.- Saber dar y recibir.

- Ser generoso.

- Dar y recibir valores personales: escucha, compañía y/o atención

$\mathrm{X}$.- Tener valores alternativos.

- Dar sentido a la vida.

XI.- Ser capaz de superar las dificultades y frustraciones.

- Capacidad de superarse en situaciones difíciles.

XII.- Ser capaz de integrar polaridades.

- Integrar lo cognitivo y lo emocional.

Rovira (1998), es el primer autor que ofrece subdimensiones del concepto para su medición, lo que puede mejorar los ítems del concepto inteligencia emocional.

\section{$\square$ El modelo de Vallés y Vallés.}

Vallés y Vallés (1999) describe en su modelo una serie de habilidades que componen la inteligencia emocional, enumerándolas éstas en diferentes aspectos: conocerse a sí mismo, automotivarse, tolerar la autofrustación, llegar a acuerdos razonables con compañeros y compañeras, identificar las situaciones que provocan emociones positivas y negativas, saber identificar lo que resulta importante en cada situación, autorreforzarse, contener la ira en situaciones de provocación, mostrarse optimista, controlar los pensamientos, autoverbalizarse para dirigir el comportamiento, rechazar peticiones poco razonables, defenderse de las críticas injustas de los demás mediante el diálogo, aceptar las críticas justas de manera adecuada, despreocuparse de aquello de nos podría obsesionar, ser un buen conocedor del comportamiento de los demás, escuchar activamente, valorar opiniones, prever reacciones, observar su lenguaje, valorar las cosas positivas que hacemos, ser capaz de divertirse, hacer actividades menos agradables pero necesarias, sonreír, tener confianza en sí mismo, mostrar dinamismo y actividad, comprender los sentimientos de los demás, conversar.

Asimismo el autor, establece otras características como: tener buen sentido del humor, aprender de los errores, ser capaz de tranquilizarse, ser realista, calmar a los demás, saber lo que se quiere, controlar los miedos, poder permanecer sólo sin ansiedad, formar parte de algún grupo o equipo, conocer los defectos personales y la necesidad de cambiar, tener creatividad, saber por qué está emocionado, comunicarse eficazmente con los demás, comprender los puntos de vista de los demás, identificar las emociones de los demás, autopercibirse según la perspectiva de los demás, responsabilizarse de su comportamiento, adaptarse a nuevas situaciones y, autopercibirse como una persona emocionalmente equilibrada.

Este modelo recoge, a modo de revisión bibliográfica, las características de los modelos anteriores, aunque completo en sí mismo, existe una falta de concreción en el diseño.

\section{$\square$ El modelo Secuencial de Autorregulación Emocional.}

Bonano (2001) fundamenta su modelo en los procesos de autorregulación emocional del sujeto para afrontar la emocionalidad de modo inteligente. El autor señala tres categorías generales de actividad autorregulatoria:

- Regulación de Control. Son los comportamientos automáticos e instrumentales dirigidos a la inmediata regulación de respuestas emocionales.

- Regulación Anticipatoria. Anticipar los futuros desafíos que se pueden presentar. 
- Regulación Exploratoria. Adquirir nuevas habilidades o recursos para mantener nuestra homeostasis emocional.

Bonano (2001) establece que todos los seres humanos portamos un grado de inteligencia emocional, que se ha de autorregular para su eficiencia.

$\square$ El modelo Autorregulatorio de las experiencias Emocionales.

Higgins et al. (1999), fundamentan la autorregulación emocional, al igual que ocurre con Bonano pero estableciendo los siguientes procesos:

- Anticipación regulatoria. Tratar de anticipar placer o malestar futuro.

- Referencia regulatoria. Adoptar un punto de referencia positivo o negativo ante una misma situación.

- Enfoque regulatorio. Estados finales deseados: aspiraciones y autorrealizaciones (promoción) contra responsabilidades y seguridades (prevención).

Este modelo es un continuo del anterior que matiza diferentes procesos como la planificación emocional.

$\square$ El modelo de procesos de Barret y Gross.

Dichos autores, en 2001 y a partir de los anteriores modelos, incluyen los siguientes procesos:

- Selección de la situación. Aproximación o evitación de cierta gente, lugares u objetos con el objetivo de influenciar las propias emociones.

- Modificación de la situación. Adaptarse para modificar su impacto emocional.

- Despliegue atencional. Elegir la parte de la situación en la que presta atención.

- Cambio cognitivo. Posibles significados que se eligen en una situación.

- Modulación de la respuesta. Influenciar las tendencias de acción.

Barret y Gross (2001) generan nuevos procesos a partir de Higgins et al. (1999) y completan el modelo anterior.

Los modelos tratados en este artículo sobre inteligencia emocional no ha alcanzado un elevado nivel de consenso conceptual en la comunidad científica (Vallés, 2005). Asimismo, tampoco se han desarrollado técnicas satisfactorias para medir los diferentes constructos sobre inteligencia emocional, de forma objetiva y fiable.

\section{6.- PROPUESTA DE MODELO.}

El modelo planteado se basa en aspectos, tanto internos como externos. Lo aspectos internos son características idiosincrásicas del individuo. Mientras que los aspectos exógenos o externos son comportamientos a partir de la adaptación o adopción del entorno. Cuando hablamos de entorno nos referimos a cualquier aspecto significativo derivado de otro individuo, empresa o situación.

En este sentido, las características intrínsecas del ser humano, no necesariamente han de ser innatas sino que pueden ser adquiridas mediante el aprendizaje y/o conocimiento. De este modo, entre las características principales endógenas, tanto innatas como aprendidas, están la responsabilidad, el sentido común, la voluntad y la capacidad de aprender.

Por otro lado, existen aspectos exógenos como son la habilidad de crear modelos mentales y la capacidad para adaptarse al entorno la empatía, la capacidad para relacionarse, la capacidad para comunicarse y la persuasión.

A continuación, se han explicado cada uno de las dimensiones:

\section{$\square$ Dimensiones endógenas (internas).}

- Responsabilidad. Se entiende responsabilidad como la capacidad de un individuo de entender, comprender las consecuencias que pueden llevar las acciones en un futuro intuido.

- Sentido común. Capacidad de planificar, organizar y controlar aspectos, de forma responsable. Dicha capacidad está intrínsecamente relacionada con la anterior.

- Voluntad. Habilidad de los individuos para seguir obteniendo resultados, aunque éstos no sean positivos.

- Capacidad de aprender. Habilidad del individuo para imitar comportamientos y acciones, pudiendo interpretarlas y modificarlas, a partir de una base de aprendizaje.

\section{$\square$ Dimensiones exógenas (externas)}

- Habilidad de crear modelos mentales. Capacidad de los individuos de crear modelos, a partir de sus experiencias con el entorno, con el fin de no volver a utilizar, otra vez todos los recursos utilizados, en primera instancia, ya que la nueva situación puede estar incrustada de forma ge- 
neral en los modelos mentales preestablecidos.

- Persuasión. Habilidad para influir en comportamientos ajenos al nuestro.

- Capacidad para adaptarse al entorno. Habilidad de un individuo de asumir pautas de conductas, a partir de la observación, con la finalidad de adaptarse a diferentes situaciones, sin que ello signifique una introducción fuera de lo que establece el grupo como normal.

- Empatía. Se entiende como la capacidad de un individuo de ponerse en el lugar de otra persona. En este sentido, dicha capacidad es importante porque puede hacer que el individuo se dé cuenta de lo que desea y expresa; no con palabras, otro individuo. Ello puede generar, mediante un sentimiento de conocimiento estrategias de desarrollo de nuestra inteligencia emocional.

- Capacidad para relacionarse. Esta capacidad está relacionada con la habilidad de un individuo para establecer lazos sociales con otros individuos, de forma no verbal. Asimismo, trata de las relaciones que se tienen con organizaciones, sistemas o sociedades como por ejemplo la relación que tiene un individuo con su trabajo o con su empresa.

Capacidad para comunicarse. Se explicita en la habilidad que puede tener un individuo para comunicarse con otros, o con organizaciones, sistemas o sociedades, pero de forma verbal.

\section{7.- CONCLUSIONES.}

El estudio ha determinado que existen dimensiones endógenas y exógenas del constructo inteligencia emocional. A partir de aquí, la principal aportación que tiene esta investigación es un modelo de medida del constructo inteligencia emocional. Asimismo, el modelo establece como dimensiones internas: la responsabilidad, el sentido común, la voluntad y la capacidad de aprender.

Por otro lado, las dimensiones externas son: la empatía, la capacidad para relacionarse, la capacidad para comunicarse, habilidad de crear modelos mentales, la persuasión y la capacidad para adaptarse al entorno. Otra aportación del modelo, es su extensión en su aplicación, puede utilizarse, no sólo en individuos o a nivel individual, como se exponen en modelos explicados a partir de la literatura, sino que se puede realizarse en sistemas organizativos, como empresas.

Las implicaciones a nivel educativo del modelo de inteligencia emocional realizado puede desembocar en la mejora de los sistemas de evaluación, ya que estos puede adaptarse en el aula, a partir del modelo aportado, dependiendo del desarrollo de la inteligencia emocional del grupo de alumnos. Por otro lado la inteligencia emocional, puede tener una influencia positiva en la clase y por lo tanto, mejorar la motivación e implicación, no sólo del alumno sino también del profesor, ya que este último puede observar a corto plazo resultados; objetivos fijados por el profesor, a partir de la normativa de la administración sobre curriculum y contenidos.

La principal limitación que tiene el modelo es que no existen evidencias empíricas con las cuales contrastarlo y compararlo. No obstante, en futuras investigaciones, y para solventar esta limitación, se va a proceder a realizar una escala de medición a partir de las dimensiones propuestas, el desarrollo de ítems y un estudio de campo para medir el grado de inteligencia emocional.

\section{8.- REFERENCIAS BIBLIOGRÁFICAS.}

Bar-On, R. (1988). The development of a concept of psycholoogical well-being, Unpublished doctoral dissertation, Rodhes University, South Africa.

Bar-On, R. (1997). The Emotional Quotient inventory (EQ-I): Techical Manual, Toronto, Canadá: Multi-Health Systems.

Barret, L.F. \& Gross, J.J. (2001). Emotional Intelligence. A process model of emotion representation and regulation. En T.J. Mayne y G.A. Bonano (Eds.). Emotions, Current Issues and future directions, New York: The Guilford Press.

Boccardo, F., Sasia, A.R. \& Fontenla, E.G. (1999). Inteligencia emocional. En A. Vallés, El desarrollo de la inteligencia emocional, Benacantil.

Bonano, G.A. (2001). Emotion self-regulation. En T.J. Mayne y G.A. Bonano (Eds.), Emotions, Current sigues and future directions. New Cork: The Guildford Press.

Cooper, R.K. \& Sawaf, A. (1997). Estrategia emocional para ejecutivos, Barcelona: Martínez Roca.

Elias, M., Tobias, S. \& Friedlander, B. (1999). Educar con inteligencia emocional, Barcelona: Plaza y Janés. 
Gardner, H. (1993). Multiples Intelligences. New York: BasicBooks, Traducción española en Ed. Paidós.

Goleman, D. (1995a). Emotional intelligence. New York: Bantam.

Goleman, D. (1995b). What's your emotional intelligence quotient? You'll soon find out. Utne Reader, November/December.

Goleman, D. (1998). Working with emotional intelligence, Nw York: Bantman.

Gómez Gras, J.M., Galiana Lapera, D. \& León Espí, D. (2000). "Que debes saber para mejorar tu empleabilidad". Elche: Universidad Miguel Hernández.

Higgins, E.T., Grant, H. \& Shah, J. (1999). Seft Regulation and quality of life: Emotional and non emotional life experiences. En Kahneman, Diener y Sxhwarz (Eds.), Well-being: the foundations of hedonic psychology. New York: Rusell Sage Foundation.

Martineaud, S. \& Engelhart, D. (1996). El Test de inteligencia emocional. Barcelona: Martínez Roca.

Mayer, J. D. \& Salovey, P. (1997). What is emotional intelligence? En P. Salovey \& D. Sluyter (Eds.), Emotional Development and Emotional Intelligence: Implications for Educators (pp. 331). New York: Basic Books.
Mayer, J.D. \& Cobb, C.D. (2000). Educational policy on emotional intelligence: the case for ability scales. In R. Bar-On \& J.D.A. Parker (Eds.), The handbook of emotional intelligence. San Francisco, Jossey Bass.

Mayer, J.D., Salovey, P. \& Caruso, D.R. (2000). Models of emotional intelligence, In R.J. Sternberg (Ed.), Handbook of intelligence (pp.396-420). Cambridge: Cambridge University Press.

Mehrabian, A. (1996). Manual for the Balanced Emocional Empathy Scale (BEES), Alta Mesa. Monterrey, CA: Mehrabian.

Rovira (1998). Como saber si un és emocionalment intelligent. Aloma, 2, 57-68.

Salovey, P. \& Mayer, J.D. (1990). Emotional Intelligence. Imagination, Cognition and Personality, 9, 185-211.

Vallés, A. (2005). El desarrollo de la inteligencia emocional. Benacantil.

Vallés, A. \& Vallés, C. (1999). Desarrollando la inteligencia emocional. Madrid: EOS.

Weisinger, H. (1988). Emocional intelligence at work: The untapped edge for succes, Josey-Bass, San Francisco. 